\title{
Performance assessment of polymer based electrodes for in vitro electrophysiological sensing: the role of the electrode impedance
}

\author{
Maria C. R. Medeiros*a , Ana L. G. Mestre ${ }^{\mathrm{b}, \mathrm{c}}$, Pedro M. C. Inácio ${ }^{\mathrm{b}, \mathrm{c}}$, José M.L. Santos ${ }^{\mathrm{d}, \mathrm{e}}$, Inês M. \\ Araujo $^{\mathrm{d}, \mathrm{e}}$, José Bragança $\mathrm{a}^{\mathrm{d}, \mathrm{e}}$, Fabio Biscarini ${ }^{\mathrm{f}}$ and Henrique L. Gomes ${ }^{\mathrm{b}, \mathrm{c}}$ \\ ${ }^{a}$ IT-Instituto de Telecomunicações, Departamento de Engenharia Eletrotécnica e de Computadores, \\ Universidade de Coimbra, 3030-290 Coimbra, Portugal. \\ ${ }^{\mathrm{b}}$ Universidade do Algarve, FCT, Faro, Portugal. \\ ${ }^{\mathrm{c}}$ IT-Instituto de Telecomunicações, Av. Rovisco, Pais, 1, Lisboa, Portugal. \\ ${ }^{\mathrm{d}}$ Department of Biomedical Sciences and Medicine, University of Algarve, 8005-139 Faro, \\ Portugal. \\ ${ }^{\mathrm{e}}$ Centre for Biomedical Research, CBMR, University of Algarve, 8005-139 Faro \\ ${ }^{\mathrm{f}}$ Life Science Dept. - University of Modena and Reggio Emilia ,Via Campi 103, I-41125 Modena, \\ Italy.
}

\begin{abstract}
Conducting polymer electrodes based on poly(3,4-ethylenedioxythiophene) polystyrene sulfonate (PEDOT:PSS) are used to record extracellular signals from autonomous cardiac contractile cells and glioma cell cultures. The performance of these conducting polymer electrodes is compared with Au electrodes. A small-signal impedance analysis shows that in the presence of an electrolyte, both $\mathrm{Au}$ and polymer electrodes establish high capacitive double-layers. However, the polymer/electrolyte interfacial resistance is 3 orders of magnitude lower than the resistance of the metal/electrolyte interface. The polymer low interfacial resistance minimizes the intrinsic thermal noise and increases the system sensitivity. However, when measurements are carried out in current mode a low interfacial resistance partially acts as a short circuit of the interfacial capacitance, this affects the signal shape.
\end{abstract}

Keywords: Organic bioelectronics, polymer electrodes, extracellular-signals, PEDOT:PSS

* cmedeiros@deec.uc.pt; phone +351239796 200; fax +351239796 247

\section{INTRODUCTION}

Microelectrode arrays (MEAs) are considered to be a basic platform for the development of cell-based sensors. These are substrate-integrated extracellular electrode matrices kept in contact with cells in culture ${ }^{1,2}$. MEA-based neuronal-electronic interfaces have been shown to facilitate the study of neuronal network processes, effects of pharmacological drugs and mechanisms underlying pathological conditions. Recently, there is a significant effort in improving the MEA technology. These efforts have been directed at two major challenges (i) decreasing the device dimensions and (ii) improving the electrical coupling between the cell and the sensing device. Smaller devices are better for parallel activity mapping, hence the application of nanofabrication technologies in this field. Devices incorporating nanostructures have been used to record electrical activity from neurons and cardiomyocytes ${ }^{3-7}$. Often in these devices the cells spontaneously engulf protruding structures. This intimate contact between the cell and electrode improves

Organic Sensors and Bioelectronics IX, edited by loannis Kymissis, Ruth Shinar,

Luisa Torsi, Proc. of SPIE Vol. 9944, 994404 - (C) 2016 SPIE

CCC code: $0277-786 X / 16 / \$ 18 \cdot$ doi: $10.1117 / 12.2237659$

Proc. of SPIE Vol. $9944994404-1$ 
signal-to-noise ratio as compared to planar electrode systems. Another goal being pursued is the lowering of electrode impedance. The most successful strategy is the use of conducting polymer surfaces ${ }^{5,8-11}$. It has been shown that polymers offer a low impedance that facilitates signal transduction from the cell to the recording electrode ${ }^{12-16}$.

This contribution compares the performance of conventional Au electrodes with ink-jet printed polymer electrode to record extracellular signals from cells in vitro. The objective is to gain insight into the impedance parameters that control the electrical coupling between the cells and planar extracellular electrodes. The performance of the electrodes is demonstrated using signals recorded from glioma cells cultures and from clusters of autonomous cardiac contractile cells. The paper is structured as follows: First, the basic measurement system is presented in section 2. Section 3 introduces the equivalent circuit model that describes the displacement current method and how the voltage signal is related with the signal in current. Next, the impedance of metallic and polymer based electrodes is presented and discussed. Finally, the conclusions highlight the role of the individual impedance parameters in shaping the signal in current.

\section{ELECTRODES CHARACTERISTICS AND EXPERIMENTAL SETUP}

Poly(3,4-ethylenedioxythiophene) polystyrene sulfonate PEDOT:PSS electrodes were ink-jet printed on glass substrates. Printing was performed in air using a Fujifilm Dimatix Material Printer (DMP) 2831, with a DMC-11610 cartridge. Samples were annealed on a hot plate at $60^{\circ} \mathrm{C}$ for $8 \mathrm{~h}$. After annealing, ethyleneglycol (EG) was deposited on the structure by immersion and then the devices were dried in a vacuum oven at $60^{\circ} \mathrm{C}$ for $12 \mathrm{~h}$. Fig. 1 (a) shows a section of two parallel PEDOT:PSS electrodes printed on glass substrates. As test cells, we used cardiomyocytes differentiated from mouse embryonic stem cells (ESC) described elsewhere ${ }^{17,18}$ and Rat glioma C6 cells (American Type Culture Collection, ATCC). A photograph of a confluent C6 cell population on top of gold electrodes is show in Fig. 1(b). An EB with multiple foci of contractile activity is shown in Fig. 1(c). This EB has approximately 1000 cells. The sensing electrodes with cells were maintained at $37^{\circ} \mathrm{C}$ in an incubator (HERACell ${ }^{\circledR} 150$ ) with a humidified atmosphere with $5 \%$ of $\mathrm{CO}_{2}$.

All electrical measurements were performed with a Stanford low-noise current amplifier (SRS 570), or alternatively in voltage mode using the voltage amplifier (SRS 560), connected to a dynamic signal analyzer (Agilent 35670A). The low noise pre-amplifiers operated with internal batteries. Small-signal-impedance measurements were carried out by a Fluke PM 6306 impedance meter. All electrical measurements were carried out inside of a thick iron based Faraday cage to shield low frequency interferences and the entire system is mechanically decoupled from external vibrations. Fig. 1(d) shows a schematic diagram of the measuring set-up. 


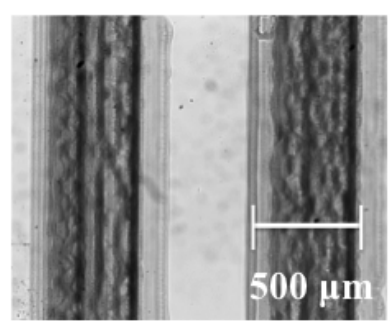

(a)

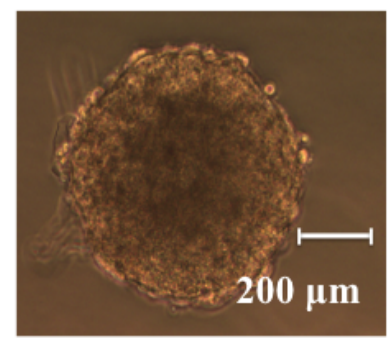

(c)

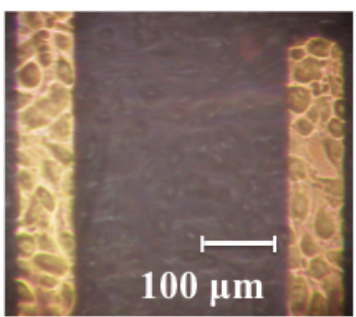

(b)

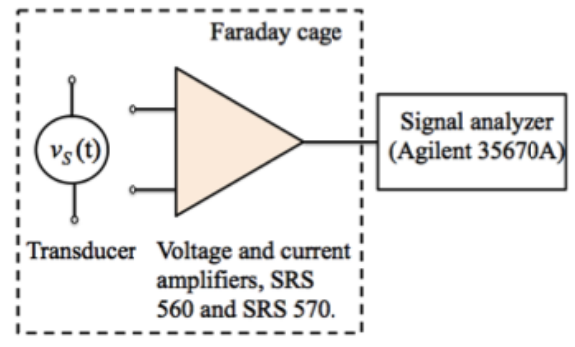

(d)

Figure 1. Cells and electrodes used. (a) section of two parallel polymer electrodes ink-jet printed on top of a glass substrate. (b) photograph of a monolayer of adherent C6 cells on top of a PET substrate with gold electrodes. (c) photograph of an EB of cardiac cells and (d) schematic diagram of the measuring set-up.

\section{EXPERIMENTAL SETUP MODEL}

Fig. 2 shows the schematic layout of the electrodes and the corresponding equivalent circuit used to interpret the data. The basic sensing system is comprised of two parallel co-planar electrodes of width $D$ and length $W$, separated by a distance $L$. One electrode acts as a sensing electrode, the other is grounded and it used as a reference electrode. When the electrodes are immersed in an electrolyte solution the ions screen the electrode potentials by forming a Helmholtz or a double-layer at the electrolyte/electrode interface. This interfacial region has been described using an equivalent circuit as depicted in Fig. 2. The double-layer is described by an interfacial resistance $\left(R_{D}\right)$ in parallel with the double-layer capacitance $\left(C_{D}\right) \cdot R_{D}$ takes into account all the possible contributions to the resistance including the charge transfer and the polarization resistance of the interface. The bulk electrolyte solution is also described by a parallel RC network takes into account the bulk electrolyte conductivity $\left(R_{S}\right)$ and capacitance $\left(C_{S}\right)$. When cells are in contact with the measuring electrode, the resistance $R_{C}$ models the signal loss between cell and the measuring electrode. The reference electrode is relatively at a long distance from the sensing electrode. Therefore, the electrical path from the cell to the reference electrode has high impedance and it is named seal impedance $\left(Z_{\text {seal }}\right)$. This simple model has been adapted from standard equivalent circuit used in literature ${ }^{19,20}$. 


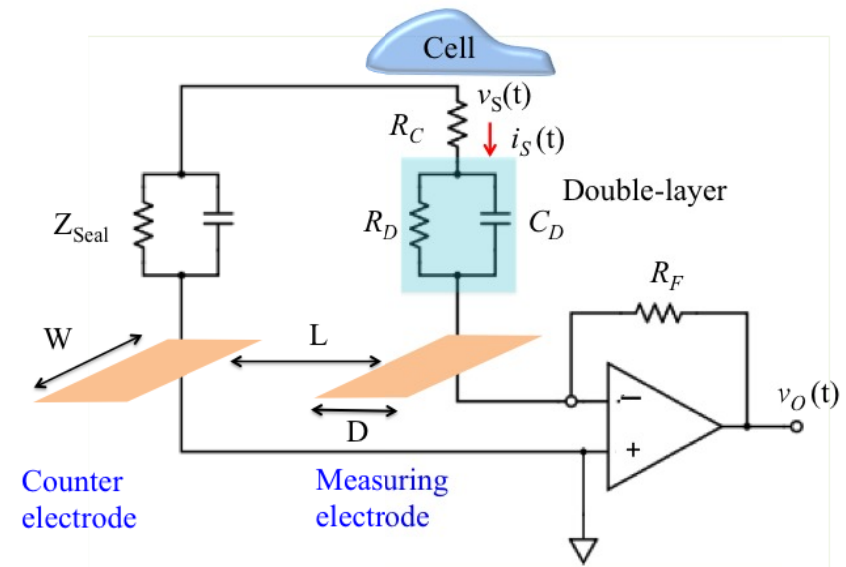

Figure 2. Schematic diagram representing the electrical coupling between the cells and the measuring circuit. A transimpedance amplifier is used. The amplified signal is $i_{s}(t) . W=3900 \mu \mathrm{m}, L=375 \mu \mathrm{m}$, and $D=550 \mu \mathrm{m}$.

\section{RESULTS}

The impedance plays a crucial role of the transducer ability to detect bioelectrical signals. The resistance generates thermal noise and basically defines the noise floor. Higher values of capacitance and low values of resistance provide better electrical coupling between cell and electrode. Therefore, it is important to measure the system impedance and understand their frequency dependence. When measuring the impedance the components that described the interfacial and the bulk electrolyte solution can be equivalent to a frequency-dependent parallel resistor $\left(R_{P}\right)$ and capacitor $\left(C_{P}\right)$. These are the parameters measured externally by an impedance analyzer. Typically, electrode/electrolyte systems behave as a two-layer system, with one interfacial layer having a high capacitance in series with a low capacitance layer. At low frequencies the high capacitive interfacial layer is probed. As the frequency increases the high capacitance is short-circuited giving rise to a transition from a high capacitance to a low capacitance that corresponds to the series sum of the interfacial and bulk capacitance. This is known as a Maxwell-Wagner relaxation. In general metallic electrodes such as $\mathrm{Au}$ immersed in cell culture medium exhibit this behavior. The frequency dependence of the capacitance $\left(C_{P}\right)$ and loss $\left(1 / \omega R_{P}\right)$ with $\omega=2 . \pi$.f for an Au electrode is shown in Fig. 3 (a). The continuous lines represent the fit using the equivalent circuit network depicted in the inset of Fig. 3 (a). The fitting is not perfect because the low frequency dispersion has not been taken into account in our model. Usually a constant phase element must be added to the equivalent circuit network to fit the dispersion at low frequencies. Fig. 3 (b) shows the frequency dependence of the polymer electrode immersed in a cell culture medium. Both the capacitance and loss are frequency dependent. However, in contrast with the gold electrodes described above, the loss rises rapidly for low frequencies, which shows that the interface has a very low resistance. The bulk electrolyte resistance $\left(R_{S}\right)$ is estimated to be approximately $1.3 \mathrm{k} \Omega$ while the interfacial double-layer resistance $\left(R_{D}\right)$ is estimated to be around $300 \Omega$. Interesting, the polymer/electrolyte system does not behave as a two-layer system and therefore does not show a Maxwell-Wagner relaxation as observed for the gold/electrolyte system. Fig. 3 (c) compares the frequency dependence of the impedance for gold and PEDOT electrodes. The impedance of the gold is several orders of magnitude higher and is strongly frequency dependent. In comparison, the impedance of the PEDOT electrodes is relatively flat upon increasing frequencies up to $1 \mathrm{MHz}$.

It is now important to understand how these differences in the impedance parameters can be explored to improve the electrode sensitivity for bioelectronics signal detection. The first obvious implication is related with thermal noise. If signals are detected and amplified as voltage, then the gold/electrolyte interface is substantially noisier than PEDOT:PSS/electrolyte interface because of its high interfacial resistance. PEDOT:PSS/electrolyte interface provide good electrical coupling between the cells and the electrodes. At low frequencies the good coupling is mainly due to the low interfacial resistance whereas the high interfacial capacitance provides a low impedance path for high frequencies. 
(a)

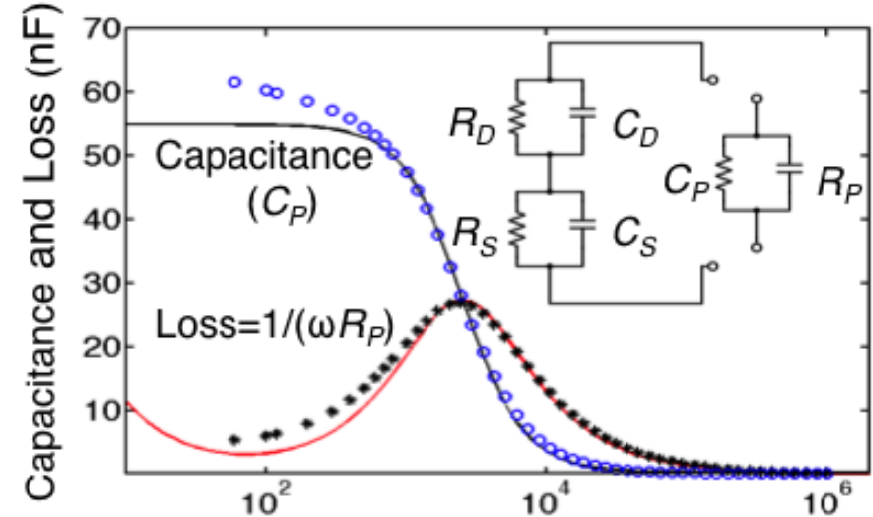

(b)

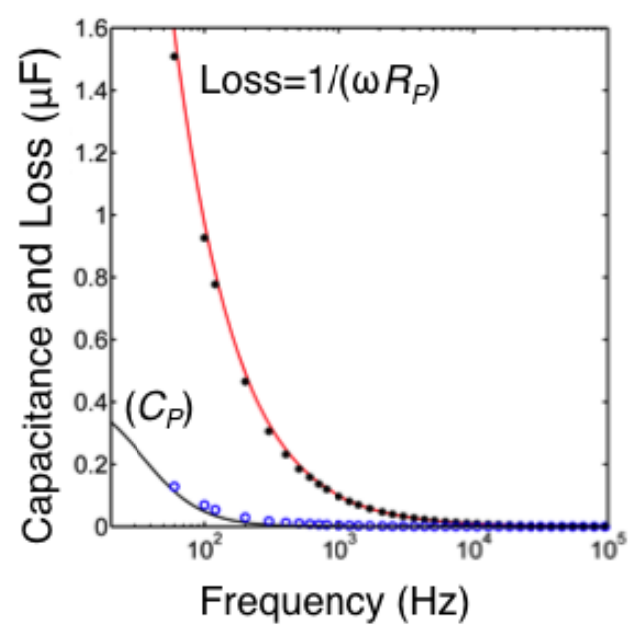

(c)

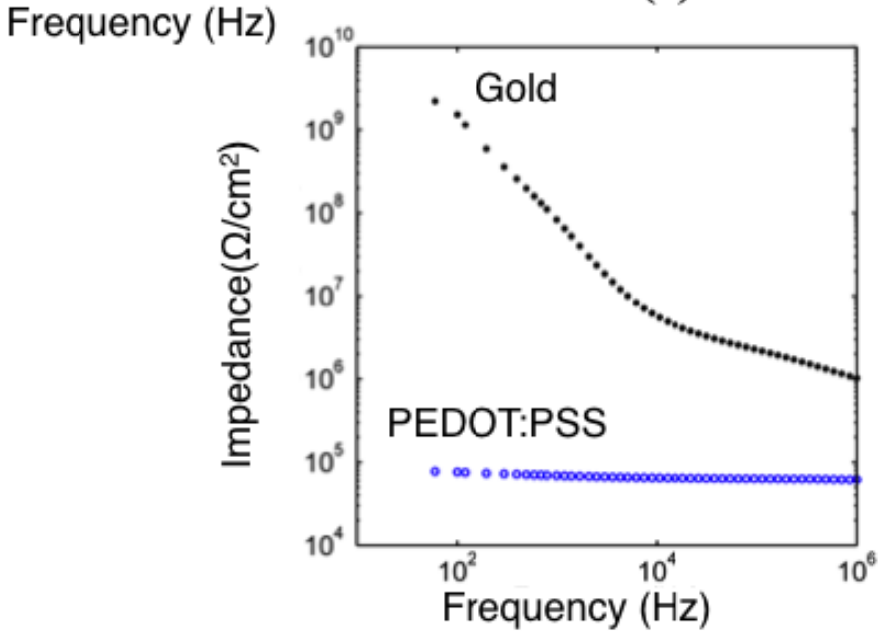

Figure 3. Comparison between the impedance of a gold electrode system with a printed PEDOT:PSS electrode. (a) Typical frequency dependence of a gold electrode system immersed in a cell culture medium. The capacitance and loss as function of the frequency has a dispersion know as Maxwell-Wagner relaxation. The inset shows the equivalent circuit used to fit the experimental data (thick lines). Fitting parameters are $R_{D}=1.4 \mathrm{M} \Omega, C_{D}=55 \mathrm{nF}, R_{S}=1.13 \mathrm{k} \Omega, C_{S}=0.36 \mathrm{nF}$. (b) The frequency dependence of the capacitance and loss for a PEDOT:PSS electrode. (c) The frequency dependence of the impedance for $\mathrm{Au}$ and for PEDOT:PSS electrodes immersed in a cell culture medium.

In our measurement setup we use a trans-impedance amplifier, whose output voltage is given by

$$
v_{o}(t)=-R_{F} i_{S}(t)
$$

where $R_{F}$ is the feedback resistance and $i_{S}(t)$ the current flowing trough the double-layer impedance. It is important to understand how this current is related with $v_{S}(\mathrm{t})$, the voltage signal generated by the cell. This relation has been derived in our previous work ${ }^{21}$.

The current $i_{S}(t)$ flowing trough the interfacial double-layer can be expressed as

$$
i_{S}(t) \cong \frac{m t}{R_{D}}+m C_{D}\left(1-e^{-t / \tau}\right)
$$


where $v_{S}(t)$ is a voltage ramp rising at constant rate $m=d v_{S}(t) / d t$.

$$
\tau=\frac{R_{D} R_{C} C_{D}}{R_{D}+R_{C}}=C_{D}\left(R_{C} / / R_{D}\right)
$$

$\tau$ is the time constant for the device to be charged or discharged.

$i_{S}(t)$ signal is the sum of two independent terms, a component proportional to $v_{S}(t)=m t$ and a transient term with a peak amplitude proportional to the product $m C_{D}$ that decays with a time constant $\tau \cong R_{C} C_{D}$.. Hence, $C_{D}$ acts as a multiplying factor for the current. Basically, a rapidly varying voltage signal produces a large transient displacement current across the capacitor. Under these conditions, the measured current signal shape is also proportional to $m$, the derivative of the original signal $v_{s}(t)$.

To understand how the signal shape is affect by the circuit components lets simulate the current response as predicted by Eq. (2) to a square voltage pulse. The simulation is represented in Fig. 4 (a). As expected at the rising and falling edges of the voltage pulse the time derivative, $d v_{S} / d t$, forces a large surge current through the capacitor giving rise to a upward and to a downward current spike. The current spike should fall down to zero when $R_{D}$ is large and to a constant DC value when $R_{D}$ is small.

The behavior described above was experimentally observed using glioma cells cultures. These cells engage into a cooperative phenomena and generate square like voltage signals with a time length that is proportional to the depth $(D)$ of the electrode. Further details can be found in a previous publication ${ }^{21}$. Fig. 4 (b) compares the current signals recorded using gold electrode with the signals recorded using a PEDOT:PSS electrode. For clarity the time trace corresponding to the PEDOT:PSS electrode was shifted along the $y y$ axis by $65 \mathrm{pA}$. Both electrodes have similar low frequency capacitance but the PEDOT:PSS electrode has a much smaller interfacial resistance $R_{D}$. This explains why the current spike recorded using the PEDOT based electrode does not return to zero, but instead to a steady state value of approximately $5 \mathrm{pA}$.

(a)

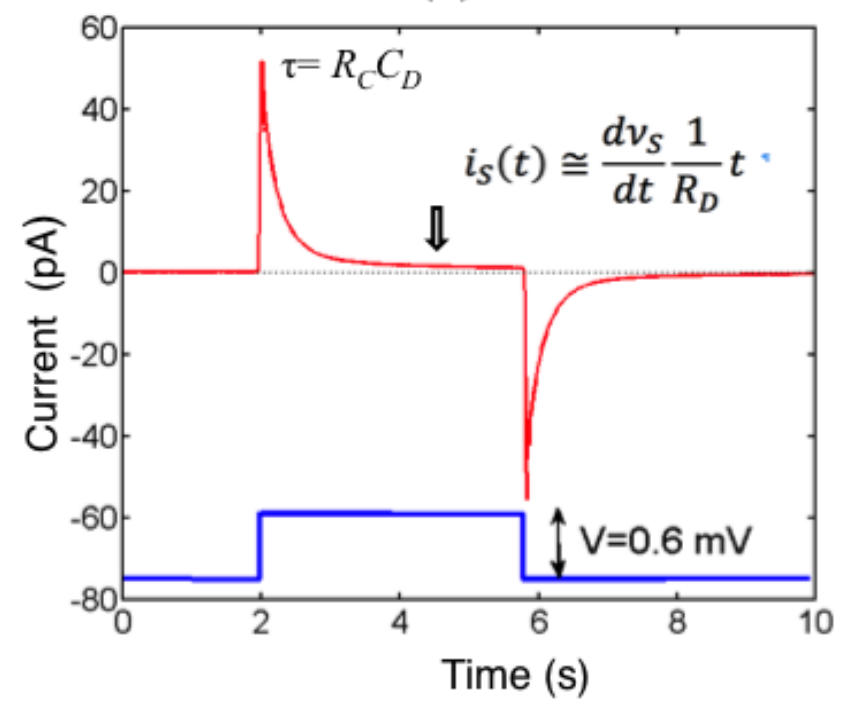

(b)

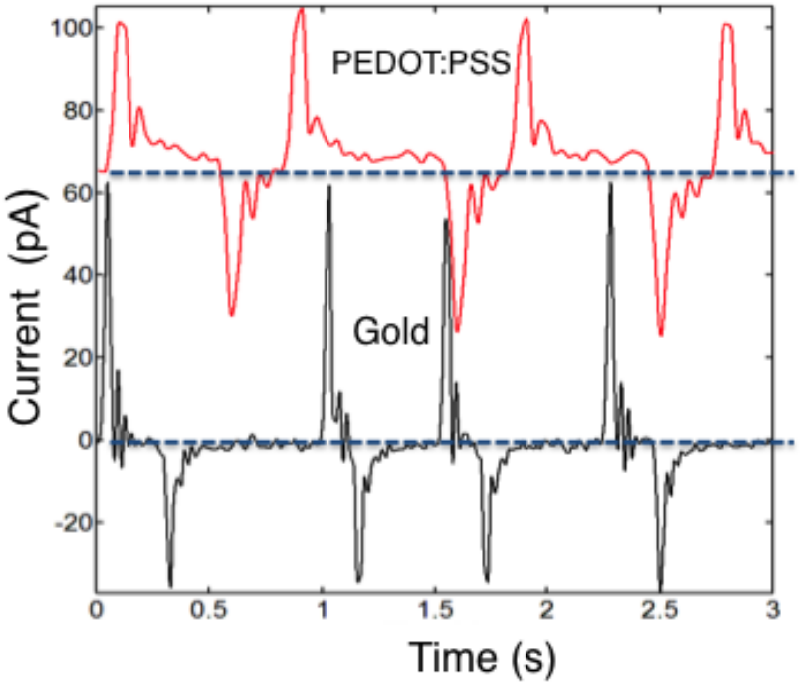

Figure 4. Signal shapes. (a) the displacement current signal in response to rectangular voltage pulse. (b) Comparison between a signal recorded using a Au electrode with a signal using a PEDOT:PSS electrode. The time trace corresponding to the PEDOT:PSS electrode was shifted along the $y y$ axis by $65 \mathrm{pA}$. The low interfacial resistance of the PEDOT:PSS electrode causes a pulse decaying to a steady state DC current. These signals have been recorded using glioma cells cultures. 
Next, we discuss how the electrode capacitance affects the displacement current signal amplitude. Two PEDOT electrodes with the same physical dimensions but with different polymer roughness and consequent different values of $C_{D}$, were used to record signals from the same cluster of contractile cells. The capacitance of one electrode system is 3.5 higher than the other. The signals recorded in the two electrode systems are shown in Fig. 5. The time trace of the high capacitance electrode is artificially shifted by $80 \mathrm{pA}$. The peak of the signal recorded in the high capacitance electrode is approximately 2.5 times higher than the signal recorded with the low capacitance electrode. The measured signal amplitude does not exactly scale with the increase in capacitance. Among the possible reasons for this discrepancy is the transfer process of the EB from one electrode to the other. After the transfer it is difficult to guarantee that the EB couples exactly in the same way to the sensing surface.

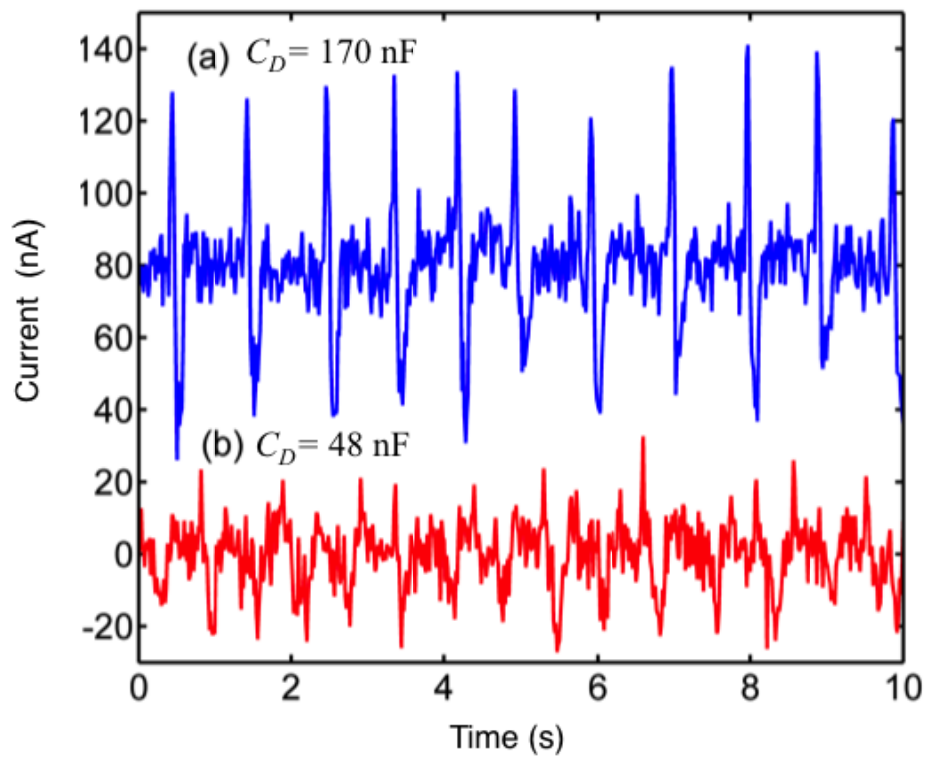

Figure 5. Comparison between two time traces of bioelectrical signals recorded from the same cluster of contractile cells using PEDOT:SS electrodes with identical dimensions but with very different interfacial capacitances. The lower time trace is the signal recorded using an electrode with $48 \mathrm{nF}$. The upper time trace is the signal recorded using an electrode with 148 $\mathrm{nF}$. For clarity, the upper time trace is artificially shifted by $80 \mathrm{pA}$.

\section{CONCLUSIONS}

PEDOT:PSS electrodes produced by ink-jet printing were used to measure extracellular bioelectrical signals in cell cultures and in cluster of contractile cells. Their electrical performance was evaluated and compared with conventional $\mathrm{Au}$ electrodes. The study of the frequency dependence of the electrode/electrolyte impedance shows that the major difference between the two electrodes is not only related with differences on the interfacial capacitance but also by a significant difference on the interfacial resistance. The PEDOT/PSS/electrolyte system has an interfacial resistance several orders of magnitude lower than the Au/electrolyte interface. A low resistance is desirable because it minimizes the intrinsic thermal noise and can be used to lower the system noise floor. However, the interfacial resistance appears in parallel with the interfacial capacitance. When the resistance is too small it can act as a leakage current path that bypass the sensing capacitance. It is shown that this effect distorts the shape of the signal when measured in current. 


\section{REFERENCES}

[1] Spira, M. E.., Hai, A., "Multi-electrode array technologies for neuroscience and cardiology," Nat. Nanotechnol. 8(2), 83-94 (2013).

[2] Obien, M. E. J., Deligkaris, K., Bullmann, T., Bakkum, D. J.,, Frey, U., "Revealing neuronal function through microelectrode array recordings," Front. Neurosci. 9(JAN), 423 (2015).

[3] Fejtl, M., Stett, A., Nisch, W., Boven, K. H.., M??ller, A., "On micro-electrode array revival: Its development, sophistication of recording, and stimulation," Adv. Netw. Electrophysiol. Using Multi-Electrode Arrays, 24-37 (2006).

[4] Blau, A., Murr, A., Wolff, S., Sernagor, E., Medini, P., Iurilli, G., Ziegler, C.., Benfenati, F., "Flexible, allpolymer microelectrode arrays for the capture of cardiac and neuronal signals," Biomaterials 32(7), 1778-1786 (2011).

[5] Furukawa, Y., Shimada, A., Kato, K., Iwata, H.., Torimitsu, K., "Monitoring neural stem cell differentiation using PEDOT-PSS based MEA,” Biochim. Biophys. Acta - Gen. Subj. 1830(9), 4329-4333, Elsevier B.V. (2013).

[6] Hierlemann, A., Frey, U., Hafizovic, S.., Heer, F., “Growing Cells Atop Microelectronic Chips: Interfacing Electrogenic Cells In Vitro With CMOS-Based Microelectrode Arrays,” Proc. IEEE 99(2), 252-284 (2011).

[7] Morin, F. O., Takamura, Y.., Tamiya, E., "Investigating neuronal activity with planar microelectrode arrays: achievements and new perspectives.," J. Biosci. Bioeng. 100(2), 131-143 (2005).

[8] Fang, Y., Li, X.., Fang, Y., "Organic bioelectronics for neural interfaces," J. Mater. Chem. C 3(25), 6424-6430, Royal Society of Chemistry (2015).

[9] Nyberg, T., Shimada, A.., Torimitsu, K., "Ion conducting polymer microelectrodes for interfacing with neural networks," J. Neurosci. Methods 160(1), 16-25 (2007).

[10] Richardson-Burns, S. M., Hendricks, J. L.., Martin, D. C., "Electrochemical polymerization of conducting polymers in living neural tissue.," J. Neural Eng. 4(2), L6-L13 (2007).

[11] Svennersten, K., Larsson, K. C., Berggren, M.., Richter-Dahlfors, A., "Organic bioelectronics in nanomedicine," Biochim. Biophys. Acta - Gen. Subj. 1810(3), 276-285, Elsevier B.V. (2011).

[12] Venkatraman, S., Hendricks, J., King, Z. A., Sereno, A. J., Richardson-Burns, S., Martin, D.., Carmena, J. M., "In vitro and in vivo evaluation of PEDOT microelectrodes for neural stimulation and recording," IEEE Trans. Neural Syst. Rehabil. Eng. 19(3), 307-316 (2011).

[13] Leleux, P., Badier, J. M., Rivnay, J., Bénar, C., Hervé, T., Chauvel, P.., Malliaras, G. G., "Conducting Polymer Electrodes for Electroencephalography," Adv. Healthc. Mater. 3(4), 490-493 (2014).

[14] Larsson, K. C., Kjäll, P.., Richter-Dahlfors, A., "Organic bioelectronics for electronic-to-chemical translation in modulation of neuronal signaling and machine-to-brain interfacing," Biochim. Biophys. Acta - Gen. Subj. 1830(9), 4334-4344, Elsevier B.V. (2013).

[15] Sessolo, M., Khodagholy, D., Rivnay, J., Maddalena, F., Gleyzes, M., Steidl, E., Buisson, B.., Malliaras, G. G., "Easy-to-fabricate conducting polymer microelectrode arrays," Adv. Mater. 25(15), 2135-2139 (2013).

[16] Khodagholy, D., Doublet, T., Quilichini, P., Gurfinkel, M., Leleux, P., Ghestem, A., Ismailova, E., Hervé, T., Sanaur, S., et al., "In vivo recordings of brain activity using organic transistors," Nat. Commun. 4, 1575 (2013).

[17] Cells, E. S., Current Protocols in Stem Cell Biology, Stem Cells 44(201) (2007).

[18] Fuegemann, C. J., Samraj, A. K., Walsh, S., Fleischmann, B. K., Jovinge, S.., Breitbach, M., "Differentiation of mouse embryonic stem cells into cardiomyocytes via the hanging-drop and mass culture methods," Curr. Protoc. Stem Cell Biol.(SUPPL.15), 1-13 (2010).

[19] Fromherz, P., "Semiconductor chips with ion channels, nerve cells and brain," Phys. E Low-Dimensional Syst. Nanostructures 16(1), 24-34 (2003).

[20] Grattarola, M.., Martinoia, S., "Modeling the Neuron-Microtransducer Junction: From Extracellular to Patch Recording," IEEE Trans. Biomed. Eng. 40(1), 35-41 (1993).

[21] Medeiros, M. C. R., Mestre, A., Inácio, P., Asgarif, S., Araújo, I. M., Hubbard, P. C., Velez, Z., Cancela, M. L., Rocha, P. R. F., et al., "An electrical method to measure low-frequency collective and synchronized cell activity using extracellular electrodes," Sens. Bio-Sensing Res. 10, 1-8, Elsevier B.V. (2016). 